\title{
$5 \mathrm{MHz} 2 \times 2$ Optical Switch in Silicon on Insulator Technology Using Plasma Dispersion Effect
}

\author{
P. Dainesi, L. Thévenaz, Ph. Robert \\ EPFL, Swiss Federal Institute of Technology, Metrology Lab. \\ 1015 Lausanne, Switzerland. (e-mail: paolo.dainesi@epfl.ch)
}

Abstract: We report on a $2 x 2$ SOI switch based on plasma dispersion effect reaching $5 \mathrm{MHz}$ of switching frequency. Measured insertion losses, extinction ratio and crosstalk at $1300 \mathrm{~nm}$ and $1550 \mathrm{~nm}$ are presented and discussed.

\begin{abstract}
Introduction
High-speed signal space switching to different physical channels is a fundamental feature for today's optoelectronic devices for telecommunications. Silicon on insulator (SOI) substrates are very promising candidates: the transparency in the infrared spectral region is very high and, in addiction, electronic devices can be integrated on the same substrate allowing the coexistence of both optical and electronic technologies, each showing its optimal features. Optical space switches based on thermooptic effect have been demonstrated in the past in SOI technology $/ 1 /$, and in silica based waveguides $/ 2 /$, but thermal switching is limited in the $100 \mathrm{KHz}$ range. Plasma dispersion effect silicon-based and SOI space switches have also been proposed in the past in mode interference $/ 3 /, / 4 /$ or total internal reflection $/ 5 /$ structures.

We report on a novel $2 \times 2$ SOI optical space switch based on the generalized Mach-Zehnder interferometer principle capable to reach $5 \mathrm{MHz}$ of switching frequency. The device is based on the plasma dispersion effect. Measurement results of fiber-to-fiber losses, extinction ratio and crosstalk at $1300 \mathrm{~nm}$ and at $1550 \mathrm{~nm}$ of the input light wavelength are reported.
\end{abstract}

\section{Device description}

The devices developed for this research are based on the well known rib waveguide structure etched in SOI wafers. An image of the cross section of such a waveguide can be found in ref. /1/, among others. The silicon dioxide layer has been obtained using the separation by implantation of oxygen (SIMOX) technology starting from a standard silicon wafer leading to a thickness of $400 \mathrm{~nm}$. The waveguide is then epitaxially grown to a height $H$ of $10 \mu \mathrm{m}$. Plasma etching of silicon to a depth of $4 \mu \mathrm{m}$ leaves $r H=6 \mu \mathrm{m}$ of unprocessed silicon. The waveguide width is $W=10 \mu \mathrm{m}$. Single mode propagation takes place for large ribs when the condition $W / H \leq 0.3+r /\left(1-r^{2}\right)^{-1 / 2}$ is fulfilled. Large ribs have also the advantage to show very low coupling losses (down to $0.35 \mathrm{~dB} /$ facet) with standard optical fibers due to the optimal matching of the fundamental fiber waveguide mode profiles.

A top view of the proposed $2 \times 2$ space switch is shown in figure 1. The principle of the generalized Mach-Zehnder interferometer is implemented through multimode interference (MMI) couplers used for light separation and recombination at the input and output ports.

The MMI couplers are designed according to the design rules reported in ref. $/ 6 /$ and $/ 7 /$ by applying the restricted multimode interference principle for paired interference. Following this principle, by placing the input waveguides to enter the $\mathrm{MMI}$ coupler at position $\pm W_{M M I} / 6$, respectively, where $W_{M M I}$ is the width of the MMl coupler, as shown in fig. 1, the length for $3 \mathrm{~dB}$ splitting can be reduced by a factor 3 . The reason is that, at that position of the input waveguides, MMI modes presenting 2, 5, 8, . zeros in the $Y$ direction will not be excited by an input field with symmetric distribution, reducing the mode phase factor as explained in detail in ref. $7 \%$. The MMI coupler lengths have been calculated this way and using the approximated approach of the effective index method. Results have then been optimized with a BPM algorithm. We have integrated two different switches with $L_{1300}=7.9 \mathrm{~mm}$ and $L_{1550}=6.6 \mathrm{~mm}$ for operation at $1300 \mathrm{~nm}$ and $1550 \mathrm{~nm}$ respectively. The total length of the two devices is $2 \mathrm{~cm}$.

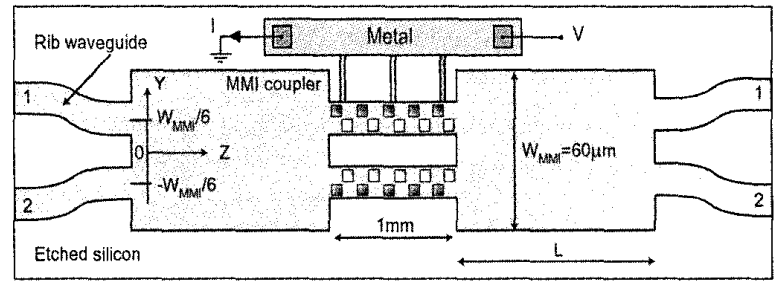

Figure 1: 2x2 Space switch. Integrated diodes doped regions connections and pads are sketched.

On a $1 \mathrm{~mm}$ long segment of the two arms of the MZI we have integrated electronic diodes in order to modulate the phase of light propagating in that segment. Charge injected through the diodes is responsible for the refractive index modulation due to plasma dispersion effect as reported in ref. $/ 8 /$, among others. Diodes doped regions are sketched in fig. 1 together with metal connections and pads. A complete description of this integration technology can be found in ref. $19 /$. When 50 to 100 diodes are connected in parallel in this configuration it is possible to obtain a $\pi$ phase shift over $1 \mathrm{~mm}$ of propagation length with a current of about $1-3 \mathrm{~mA} /$ diode. It's important to observe that diodes integrated on top of the waveguide are $100 \%$ compatible with a standard CMOS electronic integration. The waveguide etching becomes a single mask, low cost post-processing, adding optical functionality to potentially any electronic device.

Charge injection, responsible for phase modulation, is always associated to thermal power dissipation. For this reason at low modulation frequencies (typically $<300 \mathrm{KHz}$ ) thermooptic effect becomes important and can seriously reduce the device's performances by countermodulating the phase of the propagating light. At high modulation frequencies thermooptic effect is instead a simple static phase offset, which can be corrected by an adequate adjusting of the diodes operating point. 


\section{Measurement results and discussion}

Figure 2 reports the measured intensities of output ports 1 (II) and 2 (I2) when a modulating square current pulse of $300 \mathrm{~mA}$ is injected through the diodes ( $V>0$ in fig. 1). Light is injected in input port 2, output intensities are normalized to the value $I I+I 2$ when no current is injected $(V=0)$. The pulse duration is $100 \mathrm{~ns}$; the measured $10 \%$ $90 \%$ rise and fall times for $I I(12)$ are $42 \mathrm{~ns}$ (44.4 ns) and $61.5 \mathrm{~ns}$ (49.5 ns) respectively, see fig. 2 , showing the limit imposed by this technology in excess to $5 \mathrm{MHz}$.

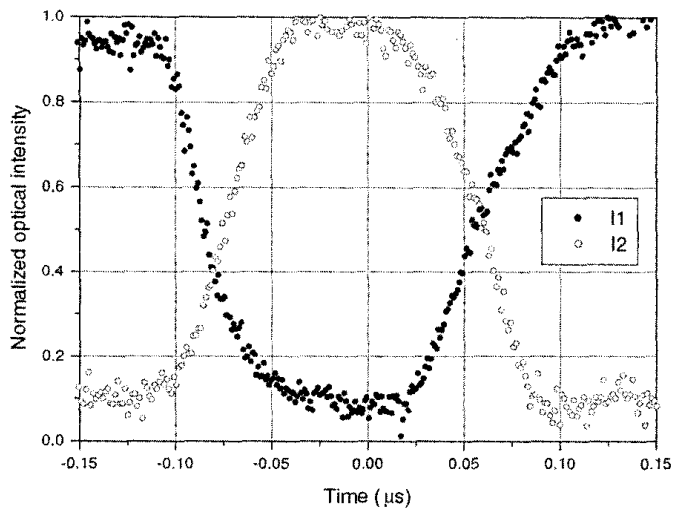

Figure 2: Intensities at output ports 1 and 2 for a $100 \mathrm{~ns}$ $300 \mathrm{~mA}$ current pulse. Diodes are forward biased.

Extinction ratio and crosstalk of about $9.5 \mathrm{~dB}$ have been measured for input light at $1300 \mathrm{~nm}$ (fig. 2). At $1550 \mathrm{~nm}$ the extinction ratio is $6.7 \mathrm{~dB}$ for 11 and $5.4 \mathrm{~dB}$ for 12 , the crosstalk $I I$ to $I 2(I 2$ to $I I)$ is $6.5 \mathrm{~dB}(5.5 \mathrm{~dB})$. All these values are comparable with those reported in ref. $/ 1 /$.

At low modulation frequencies thermooptic effect could limit seriously the performance of these devices because of the high power dissipation needed for injecting current pulses of this magnitude. We have then solved this problem by reverse biasing the diodes ( $V<0$ in fig. 1). A negative voltage drop across the diodes will cause a small current flow, resulting in a negligible plasma dispersion effect and an efficient thermooptic effect. In this way the device will use the most appropriate modulating effect in each frequency range. Modulation between $100 \mathrm{KHz}$ and $800 \mathrm{KHz}$ still remains problematic for our devices as neither effect is dominant. We have used this solution for a modulating square current pulse $8 \mu$ s long. Results are reported in figure 3. For an injected current of $50 \mathrm{~mA}$, the voltage drop across the diodes is $-9 \mathrm{~V}$. The total measured dissipated power for a $\pi$ phase shift is then $450 \mathrm{~mW}$, in good agreement with theoretical calculated values reported in ref. $/ 9 /$ and $/ 10 /$. From fig. 3 values of extinction ratio and crosstalk of about $8 \mathrm{~dB}$ can be measured. Light injected is at $1300 \mathrm{~nm}$ and similar results have been measured for light at $1550 \mathrm{~nm}$. Measured rise (heating) and fall (cooling) times for $I 1(12)$ are $1.64 \mu \mathrm{s}(1.9 \mu \mathrm{s})$ and $3.6 \mu \mathrm{s}(3.5 \mu \mathrm{s})$ respectively, limiting thermooptical switching bandwidth to about $95 \mathrm{KHz}$. These values are compatible with typical thermooptic effect characteristic times $/ 10 \%$.

Fiber-to-fiber loss measurements have been performed on both devices for the two different injected light wavelengths. The value of about $21 \mathrm{~dB}$ is far from the best reported $11 /, 14 /$. Preliminary researches performed with Fabry-Perot interference method have evidenced an absorption coefficient of $4 \mathrm{~dB} / \mathrm{cm}$ on this wafer. This value is unexpected because we have developed devices showing an absorption coefficient of $1.5 \mathrm{~dB} / \mathrm{cm}$ on wafers coming from the same batch /9/. Further research will be carried on to explicate this discrepancy.

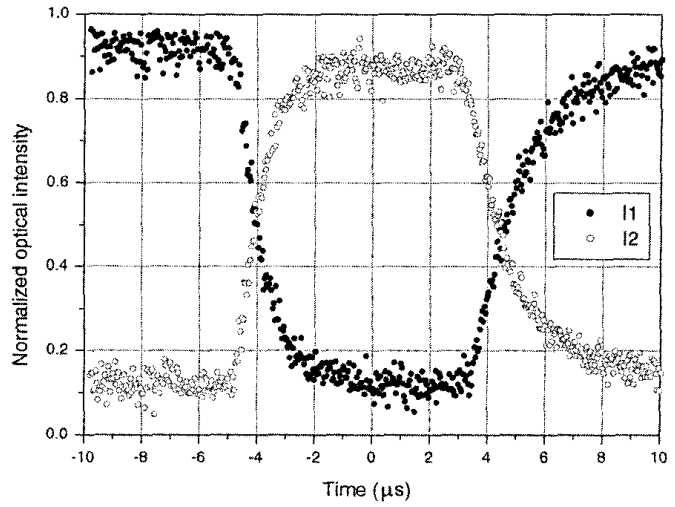

Figure 3: Intensities at output ports 1 and 2 for an $8 \mu \mathrm{s}$ $50 \mathrm{~mA}$ current pulse. Diodes are reverse biased.

While the modulation frequency is approaching its physical limit, improvements of the losses, of the extinction ratio and of the crosstalk should still be considered. We think that a significant improvement can be obtained by reducing the length of the MMI coupler and increasing the number of modes propagating through. In this way a better resolution of the self-imaged input field can improve the crosstalk between the two arms of the interferometer. Thinner wafers or lower index of refraction difference between the waveguide and the substrate are possible solutions. The last requires on the contrary higher radii in bent waveguides.

In conclusion we have presented two $2 \times 2$ optical space switches in a $100 \%$ CMOS compatible SOI technology, for $1300 \mathrm{~nm}$ and $1550 \mathrm{~nm}$ operation, using plasma dispersion effect. The modulation frequency of $5 \mathrm{MHz}$ is the highest reported to date and the devices can be also modulated at low frequency by just inverting the polarization. Losses need to be significantly reduced while extinction ratios and crosstalk are compatible with those reported in the past.

\section{references}

/1/ U. Fisher et al. Proceedings of the IEEE International SOl Conference (1995) 141-142.

2/ T. Goh et al. IEEE Photonics Technology Letters 10, 6 (1998) 810-812.

13/ J.P. Lorenzo et al. Applied Physics Letters 51, 1 (1987) $6-8$.

14/ C. Z. Zhao et al. Optics Letters 21, 20 (1996) 16641666.

15/ C. Z. Zhao et al. IEEE Photonics Technology Letters 9,8 (1997) 1113-1115

16/ M. Bachmann et al. Applied Optics 34, 30 (1995) 6898-6910.

/7/ L. B. Soldano et al. Journal of Lightwave Technology 13,4 (1995) 615-627.

/8/ R. A. Soref et al. IEEE Journal of Quantum Electronics QE-23, 1 (1987) 123-129.

19/ P. Dainesi et al. IEEE Photonics Technology Letters $12,6(2000) 660-662$

$/ 10 / \mathrm{U}$. Fisher et al. Electronic Letters 30,5 (1994) 406 408. 